\title{
Heterologous Expression of Poplar WRKY18/35 Paralogs in Arabidopsis Reveals Their Antagonistic Regulation on Pathogen Resistance and Abiotic Stress Tolerance via Variable Hormonal Pathways
}

\author{
Li Guo ${ }^{1,2}$, Chaofeng $\mathrm{Li}^{1,3}{ }^{\mathbb{C}}$, Yuanzhong Jiang ${ }^{1,4}$, Keming Luo ${ }^{1, *}$ and Changzheng $\mathrm{Xu}^{1, *}$ \\ 1 Chongqing Key Laboratory of Plant Resource Conservation and Germplasm Innovation, School of Life \\ Sciences, Southwest University, Chongqing 400715, China; g11231@yeah.net (L.G.); \\ lichaofeng.1987@163.com (C.L.); jyz88623@126.com (Y.J.) \\ 2 Crop Functional Genomics, Institute of Crop Science and Resource Conservation (INRES), University of \\ Bonn, 53113 Bonn, Germany \\ 3 Asian Natural Environmental Science Center, The University of Tokyo, 1-1-8 Midori-cho, Nishitokyo, \\ Tokyo 188-0002, Japan \\ 4 Key Laboratory for Bio-resources and Eco-environment of Ministry of Education, College of Life Science, \\ Sichuan University, Chengdu 610065, China \\ * Correspondence: kemingl@swu.edu.cn (K.L.); xucz@swu.edu.cn (C.X.); \\ Tel.: +86-23-6825-3021 (K.L.); Fax: +86-23-6825-2365 (K.L.)
}

Received: 3 June 2020; Accepted: 28 July 2020; Published: 30 July 2020

\begin{abstract}
WRKY transcription factors (WRKY TFs) are one of the largest protein families in plants, and most of them play vital roles in response to biotic and abiotic stresses by regulating related signaling pathways. In this study, we isolated two WRKY TF genes PtrWRKY18 and PtrWRKY35 from Populus trichocarpa and overexpressed them in Arabidopsis. Expression pattern analyses showed that PtrWRKY18 and PtrWRKY35 respond to salicylic acid (SA), methyl JA (MeJA), abscisic acid (ABA), B. cinereal, and $P$. syringae treatment. The transgenic plants conferred higher $B$. cinerea tolerance than wild-type (WT) plants, and real-time quantitative (qRT)-PCR assays showed that PR3 and PDF1.2 had higher expression levels in transgenic plants, which was consistent with their tolerance to $B$. cinereal. The transgenic plants showed lower $P$. syringae tolerance than WT plants, and qRT-PCR analysis (PR1, PR2, and NPR1) also corresponded to this phenotype. Germination rate and root analysis showed that the transgenic plants are less sensitive to $\mathrm{ABA}$, which leads to the reduced tolerance to osmotic stress and the increase of the death ratio and stomatal aperture. Compared with WT plants, a series of ABA-related genes (RD29A, ABO3, ABI4, ABI5, and DREB1A) were significantly down-regulated in PtrWRKY18 and PtrWRKY 35 overexpression plants. All of these results demonstrated that the two WRKY TFs are multifunctional transcription factors in plant resistance.
\end{abstract}

Keywords: PtrWRKY18; PtrWRKY35; multifunctional; pathogen resistance; water-deficit

\section{Introduction}

Thanks to their sessile lifestyle, high plants have to face various biotic and abiotic stresses [1]. To cope with these challenges, plants have evolved sophisticated mechanisms to perceive these environmental stresses and respond optimally [2]. The activation of defense or acclimation machinery plays important roles in preventing further damages to the entire plant. The signals that participate in plant responses are usually divided into two types: fast-moving signals that react within minutes, 
such as methyl JA (MeJA) or methyl SA (MeSA), and slow-moving signals that need several hours to transport and respond to changes, such as jasmonic acid (JA), salicylic acid (SA), or azelaic acid [3].

Recognition and transduction of stress signals to activate plant responses and regulation of stress-resistant genes are the key steps for enabling stress tolerance in plants. The stress-resistant genes are mainly induced at the transcriptional level, and many transcription factor (TF) families such as WRKY, AP2 (APETALA2)/ERF (ethylene responsive factor), and NAC (NAM, ATAF1/2, CUC1/2) play crucial roles in activating the expression of many stress-resistant genes during diverse biotic and abiotic stress responses [4].

WRKY transcription factors (WRKYs) belong to one of the largest TF families in plants, which are named by the highly conserved DNA-binding region WRKY domain (the WRKYGQK motifs at the N-terminal and a zinc finger motif at the C-terminus) [5]. Depending on the numbers of WRKY domain and the features of zinc-finger motif, WRKYs are divided into three groups [6,7]: Group I (containing two WRKY domains and one zinc-finger-like motif C2H2), Group II (containing one WRKY domain and one $\mathrm{C} 2 \mathrm{H} 2$ zinc-finger-like motif), and Group III (containing one WRKY domain and one C2HC zinc-finger-like motif [8].

Increasing evidence demonstrates that WRKYs play vital roles in pathogen defense, and are regulated by elicitors such as wounding, SA, and JA. For example, CaWRKY2 and PtrWRKY40 play roles in resistance to pathogens [9,10]. AtWRKY25 is involved in plant defense against Pseudomonas syringae (P. syringae) [11], while AtWRKY3 and AtWRKY4 enhance the defense against Botrytis cinerea (B. cinereal) [12]. WRKYs also participate in plant responses to abiotic stresses and abscisic acid (ABA) signaling [13]. In Arabidopsis, the knock-out of $A B O 3$ encoding a WRKY transcription factor decreased the ABA sensitivity, but increased the sensitivity to drought stress [14]. AtWRKY18, AtWRKY40, and AtWRKY60 have been found to not only function in plant responses to pathogen defense, but also regulate the response to drought stress $[15,16]$. In rice, the expressions of OsWRKY24, OsWRKY51, OsWRKY71, and OsWRKY72 are induced by ABA [17]. The soybean GmWRKY13, GmWRKY21, and GmWRKY54 improve the abiotic stress tolerance in transgenic Arabidopsis. Moreover, WRKYs also play roles in seed development and germination [18], leaf senescence [19], and secondary metabolism [20].

We previously identified paralogous PtrWRKY18 and PtrWRKY35 encoding the homologs of Arabidopsis WRKY18, WRKY40, and WRKY60 in poplar [21]. Functional characterization revealed that both WRKY paralogs redundantly regulate the defense against biotrophic pathogen and SA-mediated signaling pathway [22]. In this study, we heterologously expressed the poplar WRKY18 and WRKY35 in Arabidopsis, and found their differential regulation on pathogen resistance and abiotic stress tolerance. Our results indicated that these WRKY transcription factors modulate different hormonal signaling to confer multiple biotic and abiotic stress responses.

\section{Results}

\subsection{The Expression of PtrWRKY18 and PtrWRKY35 Is Induced by Various Hormones and Biotic Stresses}

To comprehensively understand the role of PtrWRKY18 and PtrWRKY35 in stress response, the GUS ( $\beta$-glucuronidase) reporter lines driven by their promoter fragments were firstly generated in Arabidopsis. The homozygous transgenic plants with single copy insertion were exposed to various hormones and fungal pathogens, and determined by histological staining (Figure 1). The expression of PtrWRKY18 and PtrWRKY35 was significantly induced by P. syringae and B. cinerea, two pathogen species, as well as by SA and JA, two hormones involved in pathogen resistance (Figure 1A). In contrast, we found that the expression of both WRKY paralogs was largely repressed under ABA treatment for $4 \mathrm{~h}$ (Figure 1B), which was validated by time-course quantification of GUS activity (Figure 1C). The differential responses of the WRKY expression to hormones and pathogens implicate their variable roles in stress resistance. 
A

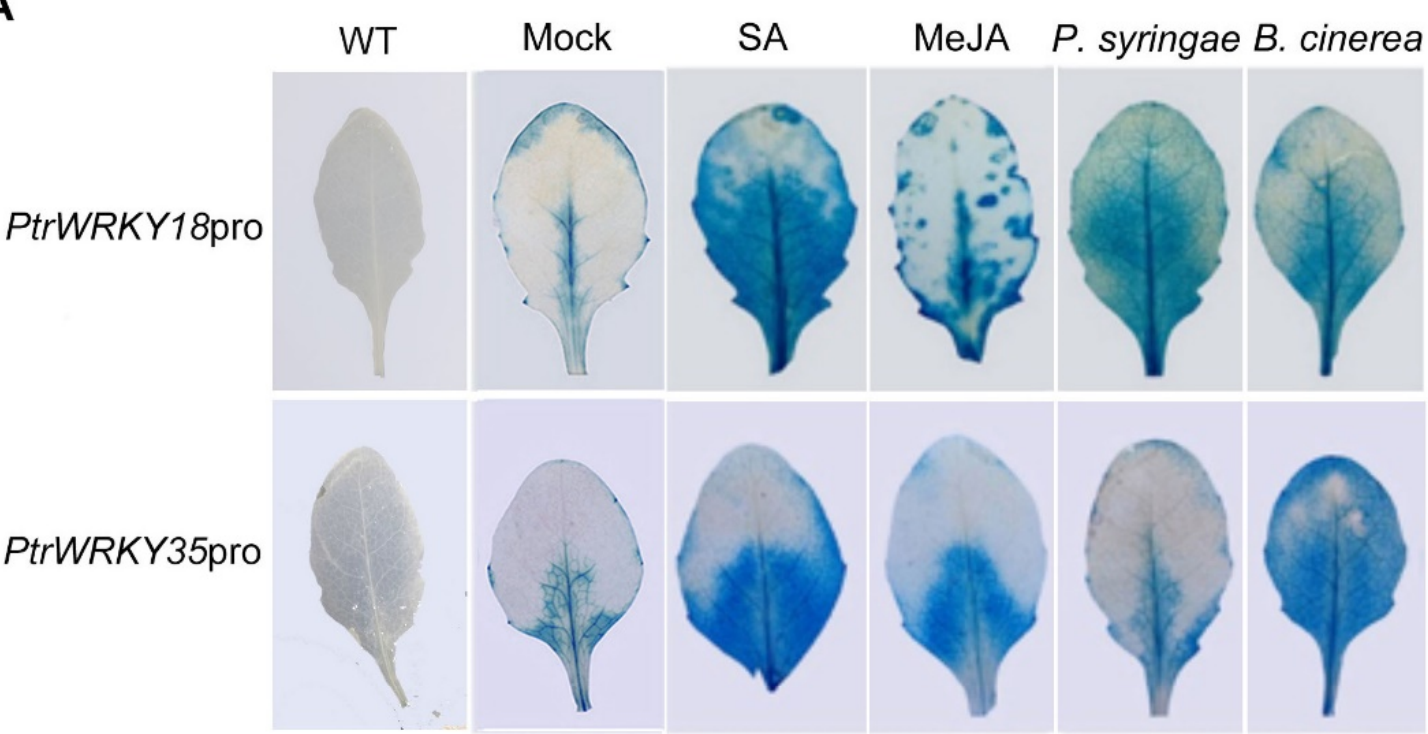

B

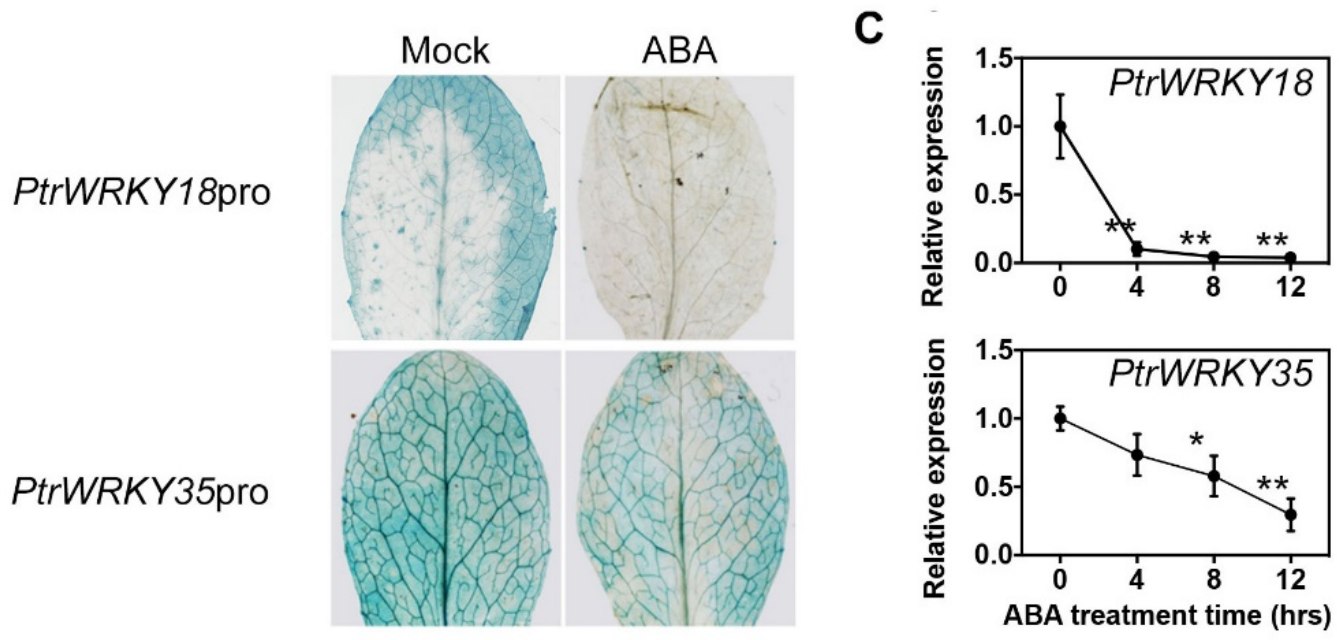

Figure 1. Expression analysis of PtrWRKY18 and PtrWRKY35. (A) GUS ( $\beta$-glucuronidase) staining of leaves of transgenic Arabidopsis overexpressing PtrWRKY18pro: GUS and PtrWRKY35pro: GUS after treated with SA, JA, B. cinerea, and P. syringae. Wild-type (WT) leaves and the transgenic leaves under mock treatment were used as negative control. (B) GUS staining of leaves of transgenic Arabidopsis overexpressing PtrWRKY18:GUS and PtrWRKY35:GUS after being treated with ABA for $4 \mathrm{~h}$. (C) Time-course quantification of GUS activities under abscisic acid (ABA) treatment. Five biological replicates were determined for each timepoint. The values for $0 \mathrm{~h}$ were normalized to 1 , and bars represent $\mathrm{SD}$. Asterisks indicate significant differences with respect to the value for $0 \mathrm{~h}$ ((Student's $t$-test: $\left.{ }^{*} p<0.05 ;{ }^{* *} p<0.01 ; n=5\right)$. MejA, methyl JA; SA, salicylic acid.

\subsection{PtrWRKY18 and PtrWRKY35 Play Positive Roles in Resistance Against B. cinerea}

To investigate the roles of PtrWRKY18 and PtrWRKY35, their overexpression vectors were transformed into WT Arabidopsis. Two independent homozygous lines containing a single insert with high PtrWRKY18 (L2 and L4) or PtrWRKY35 (L6 and L9) expression levels were selected by assays of semi-RT-PCR (Figure 2A) and qRT-PCR (Figure 2B), respectively, and used for further analyses.

The persistent expression of disease resistance genes can affect plant growth and development [23], hence we observed and compared the growth of WT and transgenic Arabidopsis. There was no significant difference between the two-week-old transgenic and WT seedlings in culture dishes (Figure 2B). Further, the seedlings were transferred into soil, and the 30-day-old WT seedlings were found to be slightly larger than the transgenic seedlings (Figure 2C). 
A

A

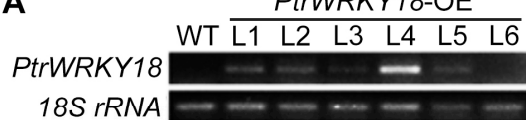

B

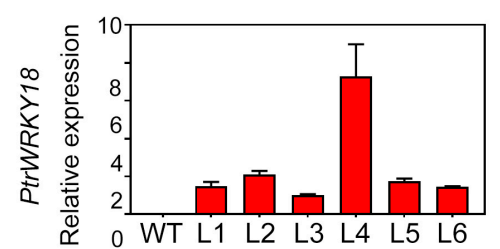

PtrWRKY35-OE

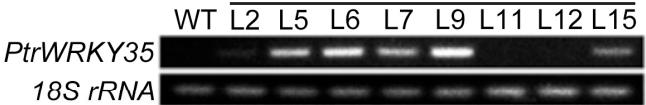

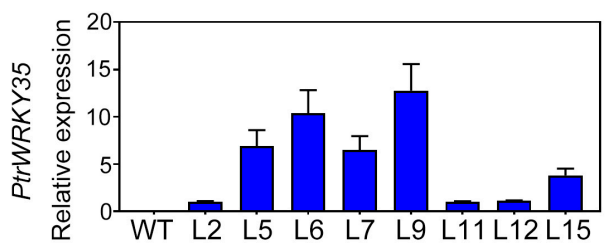

C

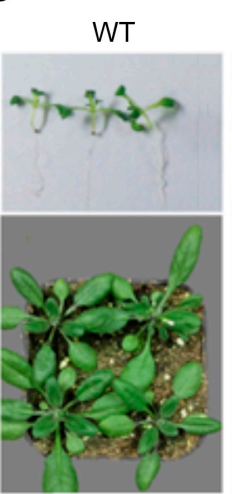

$\frac{\text { PtrWRKY18-OE }}{\mathrm{L} 2}$

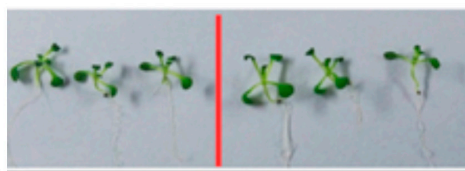

$\frac{\text { PtrWRKY35-OE }}{\text { L6 }}$

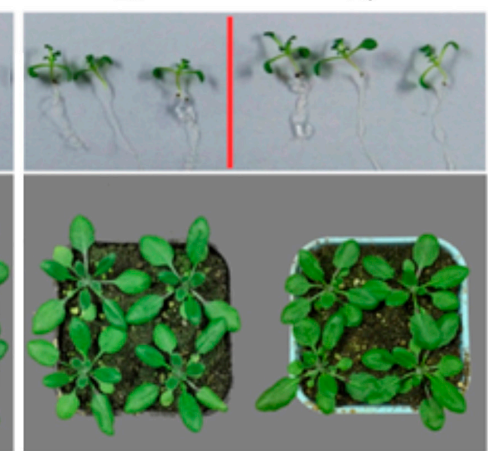

D

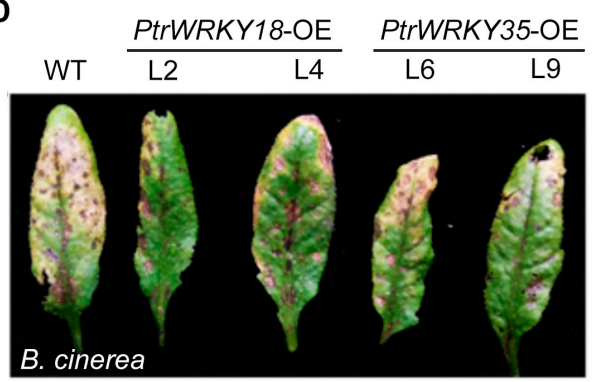

E

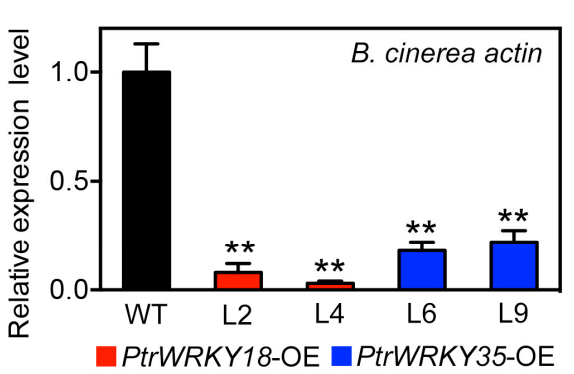

$\mathbf{F}$

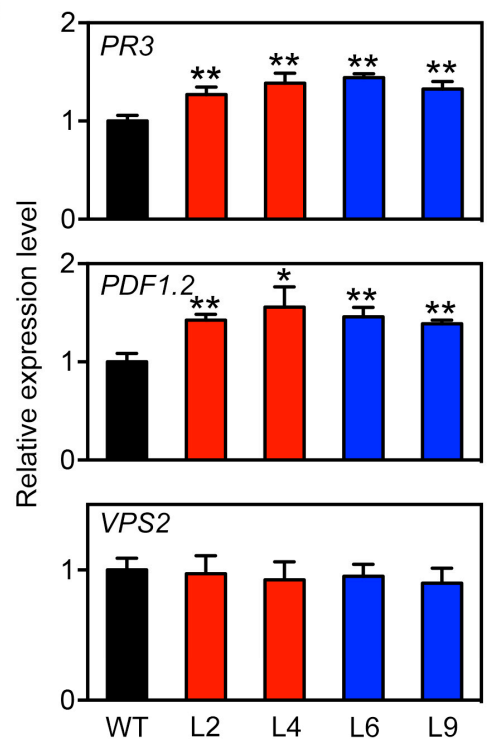

口PtrWRKY18-OE —PtrWRKY35-OE

Figure 2. Constitutive expressing PtrWRKY18 or PtrWRKY35 enhanced the Botrytis cinerea tolerance of Arabidopsis. (A,B) Semi real-time quantitative (qRT)-PCR (A) and qRT-PCR (B) analysis of transgenic Arabidopsis lines overexpressing PtrWRKY18 or PtrWRKY35. (C) Phenotype observation of two-week-old and one-month-old transgenic and WT plants. (D) Leaves phenotypes of WT, PtrWRKY18, and PtrWRKY35 overexpressed plants after B. cinerea inoculation. (E) Expression levels of Bcactin (B. cinerea actin gene) in infected plants. (F) Expression levels analysis of JA response marker genes in WT and plants overexpressing PtrWRKY18 and PtrWRKY35. PR3: pathogenesis-related gene 3; PDF1.2: plant defensin genev1.2; VSP2: vegetative storage protein gene 2 . For $(\mathbf{E}, \mathbf{F})$, average values from three biological replicates were shown. The error bars are used to represent $\mathrm{SD}\left({ }^{*} p<0.05 ;{ }^{* *} p<0.01\right)$.

In Arabidopsis, AtWRKY18, AtWRKY40, and AtWRKY60 have been proven to function in defense of plants against pathogens, such as B. cinerea and P. syringae [15]. In order to verify if PtrWRKY18 or 
PtrWRKY35 have similar functions, the transgenic plants overexpressing PtrWRKY18 or PtrWRKY35 were sprayed with the spore suspending of $B$. cinerea. As shown in Figure 2D, the leaves of transgenic Arabidopsis overexpressing PtrWRKY18 and PtrWRKY35 had a lesser necrosis area than that in WT leaves. To determine the growth status of $B$. cinerea in different genotypes of Arabidopsis thaliana, qRT-PCR was used to analyze the expression levels of $B$. cinerea actin gene. The results showed that the B. cinerea actin gene had lower expression levels in the transgenic plants (Figure 2E). Therefore, we deem that overexpression of PtrWRKY18 or PtrWRKY35 could improve the resistance to B. cinerea in Arabidopsis. In general, plant resistance to herbivorous insects or necrotrophic pathogens (such as B. cinerea) is achieved by activation of the JA signaling pathway. qRT-PCR showed that transcript levels of PR3 and PDF1.2 (Figure 2F), two marker genes in the JA signaling pathway [10], were significantly upregulated in transgenic plants, while VSP2 (Figure 2F) showed no difference between WT and transgenic plants.

These results demonstrated that PtrWRKY18 and PtrWRKY35 have positive roles in JA-mediated signaling against B. cinerea in Arabidopsis.

\subsection{PtrWRKY18 and PtrWRKY35 Play Negative Roles in Resistance Against P. syringae}

In our previous studies, we found PtrWRKY18 and PtrWRKY35 function in SA signaling pathway and regulated resistance to the biotrophic pathogen Melampsora in P. tomentosa [22]. To explore whether heterologous expression of PtrWRKY18 and PtrWRKY35 in Arabidopsis affects their function in the SA signaling pathway, P. syringae were inoculated on the leaves to observe their development. As shown in Figure 3A,B, after P. syringae inoculation, the leaves of transgenic plants had more severe chlorosis spot symptoms than WT plants. Chlorophyll contents measurement showed that the contents of chlorophyll in transgenic plant leaves were significantly decreased (Figure 3C). The biotrophic pathogens growth assay suggested that the number of $P$. syringae pathogen in leaves of transgenic plants (L2 and L9) was significantly more than that in WT, while there also were slightly more P. syringae pathogens in L4 and L6 than WT (Figure 3D). Gene expression levels assay shown that most marker genes in the SA signaling pathway, including PR1, PR2, and NPR1, were dramatically decreased in plants overexpressed PtrWRKY18 or PtrWRKY35 (Figure 3E). However, the expression level of PR5 was up-regulated in plants overexpressing PtrWRKY18, but significantly down-regulated in plants overexpressing PtrWRKY35 (Figure 3E). This may be as a result of the two genes functioning through not exactly the same manner.

These results indicated that overexpression of PtrWRKY18 and PtrWRKY35 led to a decrease of P. syringae resistance in Arabidopsis by downregulating the expression levels of most genes in the SA signaling pathway.

\subsection{Overexpression of PtrWRKY18 and PtrWRKY35 Reduced Sensitivity to ABA in Transgenic Arabidopsis}

As an important component of plant signaling pathways, the hormone ABA acts as a key signal for regulating a range of plant physiological processes, such as germination, seedling growth, root development, stomatal regulation, and defense to osmotic stress [24,25]. From the results of Figure 1B and 1C, we can learn that the expression levels of PtrWRKY18 and PtrWRKY35 are downregulated after ABA treatment. Therefore, we speculated that PtrWRKY18 and PtrWRKY35 might play roles in the ABA-independent signaling pathway.

To test the hypothesis, the seeds of WT and transgenic plants were treated with ABA during seedling development to observe their response to ABA. Compared with WT plants, the seeds of transgenic plants were germinated earlier and had significantly higher germination rates under the same concentration of ABA treatment (Figure 4A). There was no significant difference in the germination process (data not shown) and root length (Figure $4 \mathrm{~B}, \mathrm{C}$ ) between seeds of transgenic plants and WT in the absence of ABA. Although ABA treatment severely inhibited the root development of WT plants, the root length of transgenic plants was obviously longer than that of WT (Figure 4B,C). These results provide clues to the reduced sensitivity of ABA in PtrWRKY18 or PtrWRKY35 overexpressing plants. 
A

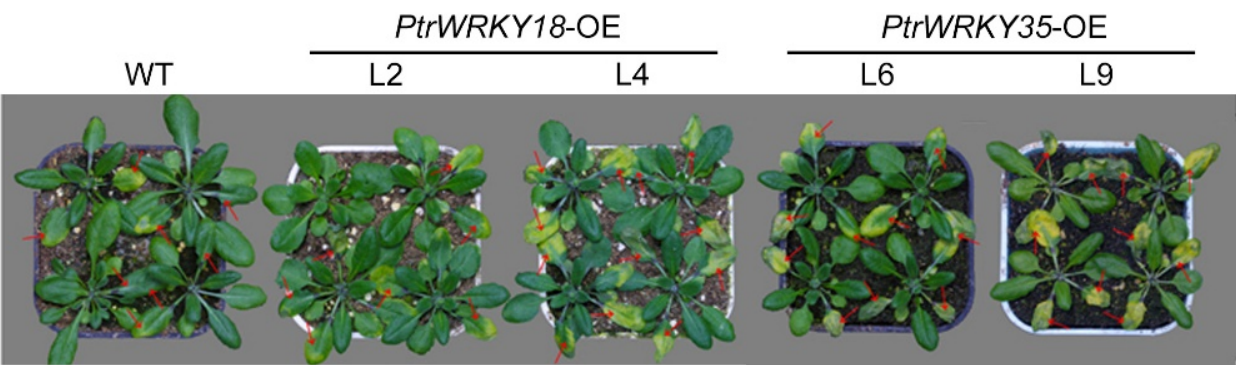

B
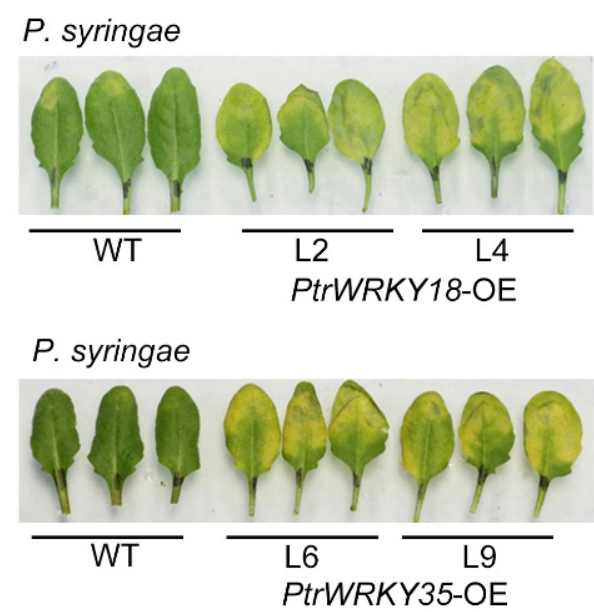

E

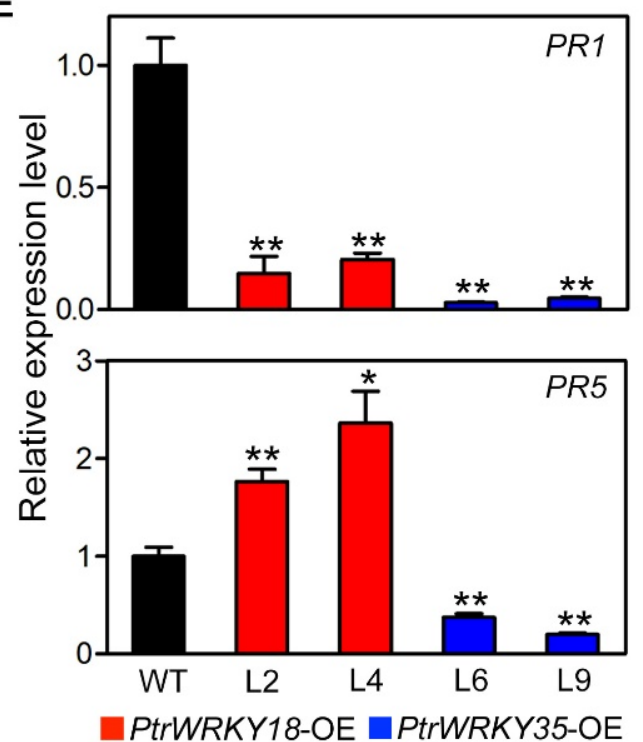

C

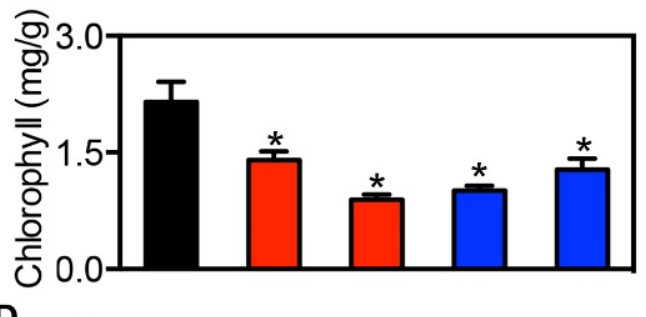$$
\text { D }
$$
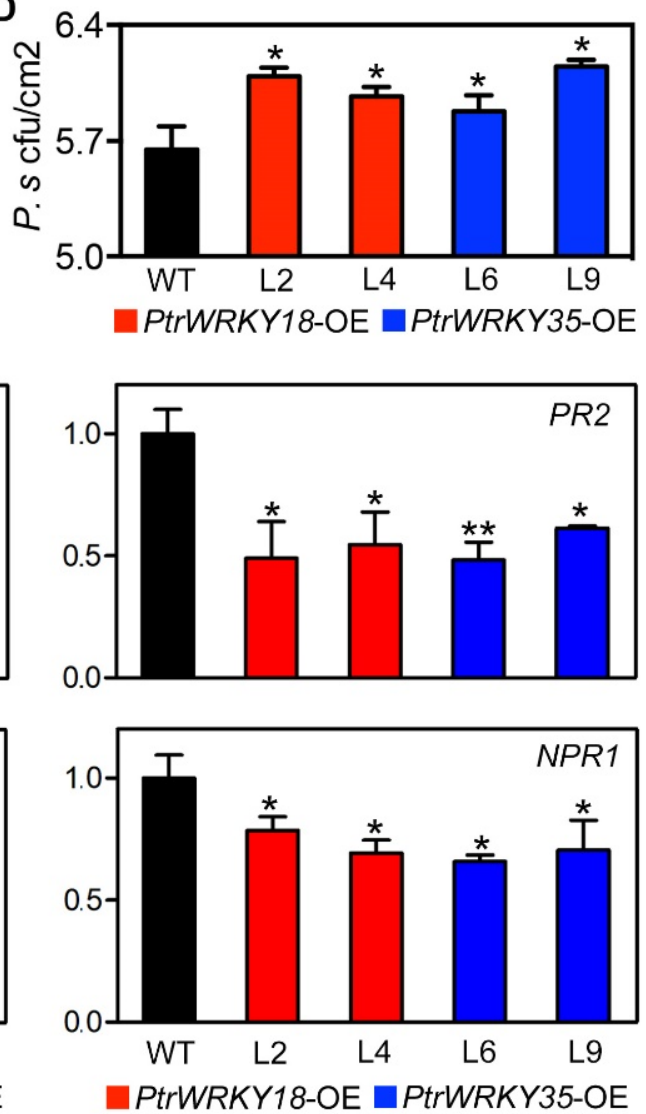

Figure 3. The Arabidopsis overexpressing PtrWRKY18 or PtrWRKY35 had decreased resistance to P. syringae. (A) Phenotype of WT and transgenic plants overexpressing PtrWRKY18 or PtrWRKY35 infected with pathogen P. syringae. (B) Leaves phenotypes of WT, PtrWRKY18, and PtrWRKY35 transgenic plants after P. syringae inoculation. (C) Quantification of total chlorophyll content in WT and transgenic plant infected with P. syringae. $\left({ }^{*} p<0.05 ; n=3\right)$. (D) Growth of P. syringae in WT and transgenic plants after inoculation $\left({ }^{*} p<0.05 ; n=3\right)$. For $\mathbf{C}$ and $\mathbf{D}$, each experiment was carried out with 20 plants and the experiment was repeated three times. (E) Expression analysis of maker genes involved in SA signaling pathway. PR1/2/5: pathogenesis-related genes 1/2/5. NPR1: non-expresser of PR genes 1 . Three repeats of biological replicates were performed for each gene, and each experiment contained three technical replicates. Values are means with $\mathrm{SD}\left({ }^{*} p<0.05 ;{ }^{* *} p<0.01\right)$. 
A
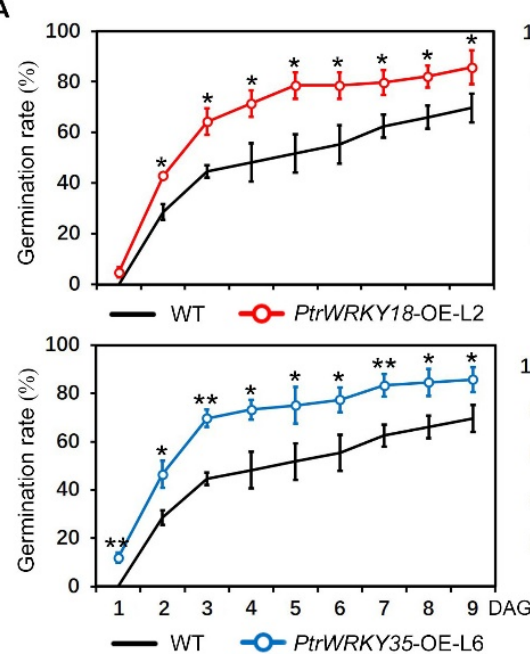

B

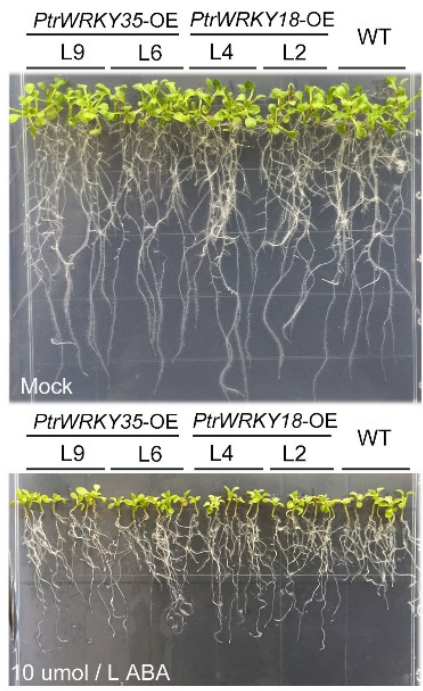

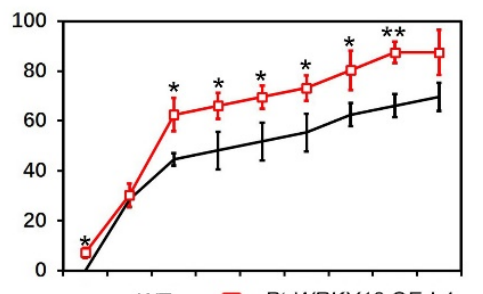

- WT $-\square$ PtrWRKY18-OE-L4

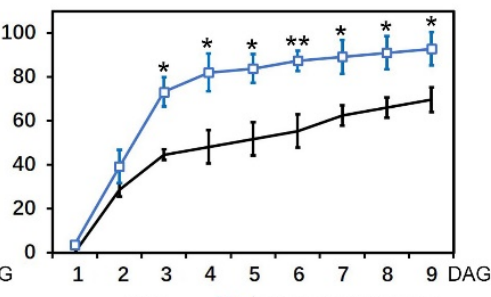

-WT $-\square-P$ trWRKY35-OE-L9
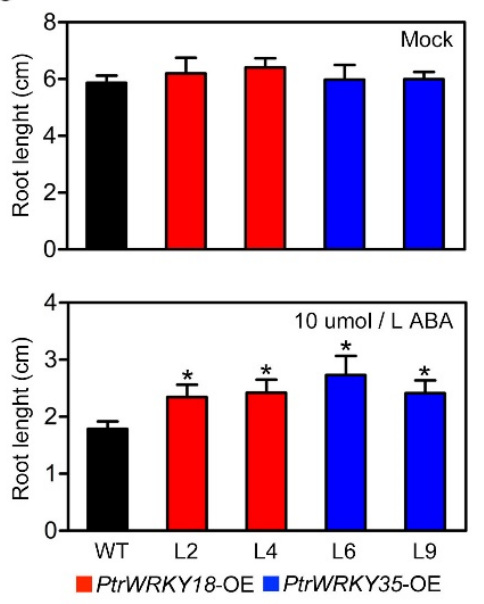

Figure 4. The Arabidopsis overexpressed PtrWRKY18 or PtrWRKY35 showed the ABA-insensitive phenotype. (A) Quantitative comparison of seeds' germination ratio between WT and plants overexpressing PtrWRKY18 or PtrWRKY35 on MS medium supplemented with ABA. Each time, the statistics contained 28 plants and three replicates were performed. The same WT line was used as control. The error bars were used to represent SD $\left(^{*} p<0.05 ;{ }^{* *} p<0.01\right)$. (B) Phenotype of root of WT and transgenic plants in MS and MS containing ABA. (C) Root length statistics of WT and transgenic plants in MS and MS containing ABA. Fifteen plants were used for each statistic, and three independent experiments were performed. SD is represented by error bars $\left({ }^{*} p<0.05\right)$.

\subsection{Overexpression of PtrWRKY18 and PtrWRKY35 Reduced Drought Tolerance in Arabidopsis}

In many plants, endogenous ABA levels dramatically accumulate in conditions of osmotic stresses, such as high salinity and drought [26,27]. The increased ABA content activates the expression of downstream transcription factors to regulate various ABA-responsive genes, so that plants can respond to osmotic stress through closing stoma, reducing transpiration, and so on $[28,29]$.

On the basis of the phenotype that transgenic plants were less sensitive to ABA (Figure 4), we hypothesized that overexpression PtrWRKY18 or PtrWRKY35 may reduce the tolerance of plants to osmotic stresses, and $\mathrm{NaCl}$ and drought treatment were used to confirm our speculation. Compared with WT, the PtrWRKY18 or PtrWRKY 35 overexpression lines were more sensitive to both high salinity and drought stress, especially the drought stress (Figure 5A). After drought treatment, transgenic plants had excessive loss of cellular water and appeared on more severe defect symptoms (Figure 5A). 
Fewer plants in transgenic plants can be revived after re-watering than the WT plants (Figure 5B). As a critical chemical messenger for osmotic response, ABA has been brought to the central stage of variation of stomatal aperture [30]. Stomata opening and closure of leaves observation showed that, for the stomatal aperture, there was no significant difference between the leaves of WT and transgenic plants in normal conditions (Figure 5C). With ABA treatment, the leaves of PtrWRKY18 or PtrWRKY35 overexpressing plants showed a larger stomatal opening than that of WT (Figure 5C). The length/width ratio of stomatal pores was usually used as an indicator of stomatal aperture to analyze the stomatal aperture in ABA-mediated drought response [31]. The statistical results showed that the guard cell of leaves from transgenic plants had a significantly larger stomatal aperture than that of WT (Figure 5D). These results suggest that PtrWRKY18 and PtrWRKY35 participated in the ABA-mediated drought response, and reduced the plants' drought tolerance via affecting the ABA sensitivity.

A
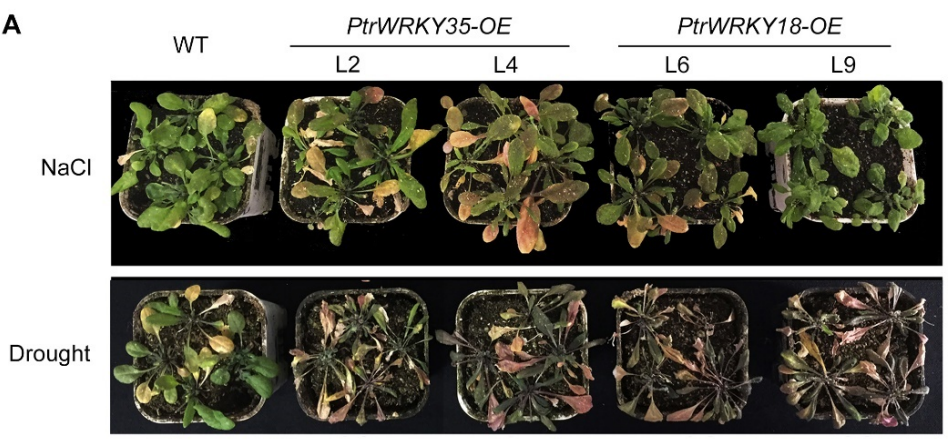

B

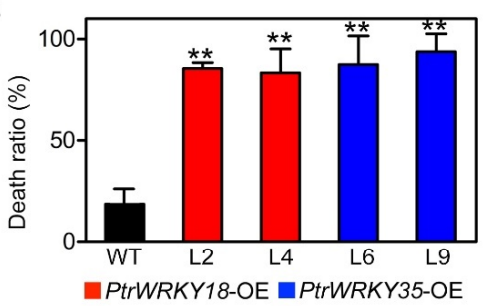

C

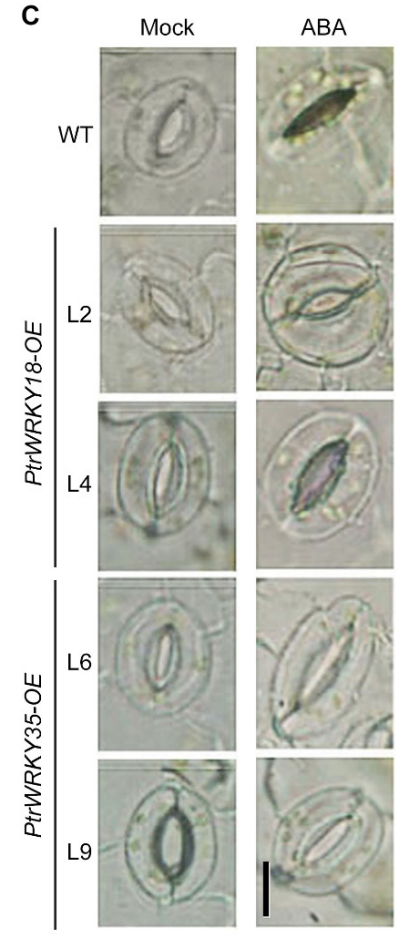

D

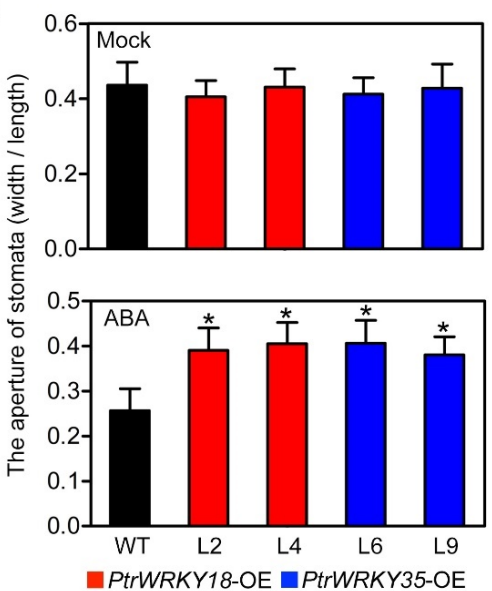

Figure 5. PtrWRKY18 and PtrWRKY35 negatively regulate plant drought defense in Arabidopsis. (A) Phenotypes of WT, PtrWRKY18, and PtrWRKY35 overexpressing plants treated with $\mathrm{NaCl}$ or natural drought. (B) Death ratio of WT and transgenic plants in (A) after re-watering. Thirty plants were used for each experiment, and three independent experiments were done. Values are means with SD $\left(^{* *} p<0.01\right)$. (C) Microscopic observation of guard cells of WT and Arabidopsis overexpressing PtrWRKY18 or PtrWRKY35 before and after ABA treatment, bars $=10 \mu \mathrm{m}$. (D) Stomatal aperture (the ratio of stomatal width to length) measurements of WT and transgenic plants before and after ABA treatment. In each experiment, 40 stomata from different plants were conducted. Three independent experiments were performed. Error bars are used to indicate $\operatorname{SD}\left({ }^{*} p<0.05\right)$. 
It has been reported that $R D 22$ and $R D 29 A$ are stress-response genes, and could be induced by salt, drought, and ABA. $A B O 3$ mediates the drought tolerance of Arabidopsis through regulating the expression of downstream genes [13]. $A B I 4$ and $A B I 5$, two ABA-insensitive genes, are inhibited by AtWRKY18, AtWRKY40, and AtWRKY60 [16]. DREB1A also plays a role in drought defense [32]. Therefore, qRT-PCR analysis (Figure 6) was used to research the mechanism of ABA-mediated drought response. As showed in Figure 6, overexpressing PtrWRKY18 or PtrWRKY35 in Arabidopsis down-regulated the expression levels of $R D 29 A, A B O 3, A B I 4, A B I 5$, and DREB1A. The results further demonstrated that PtrWRKY18 and PtrWRKY35 were involved in the signal pathway of ABA-mediated drought response. Interestingly, $R D 22$, which was the marker gene of salt, drought, and ABA stresses, displayed no significant changes in transgenic plants.
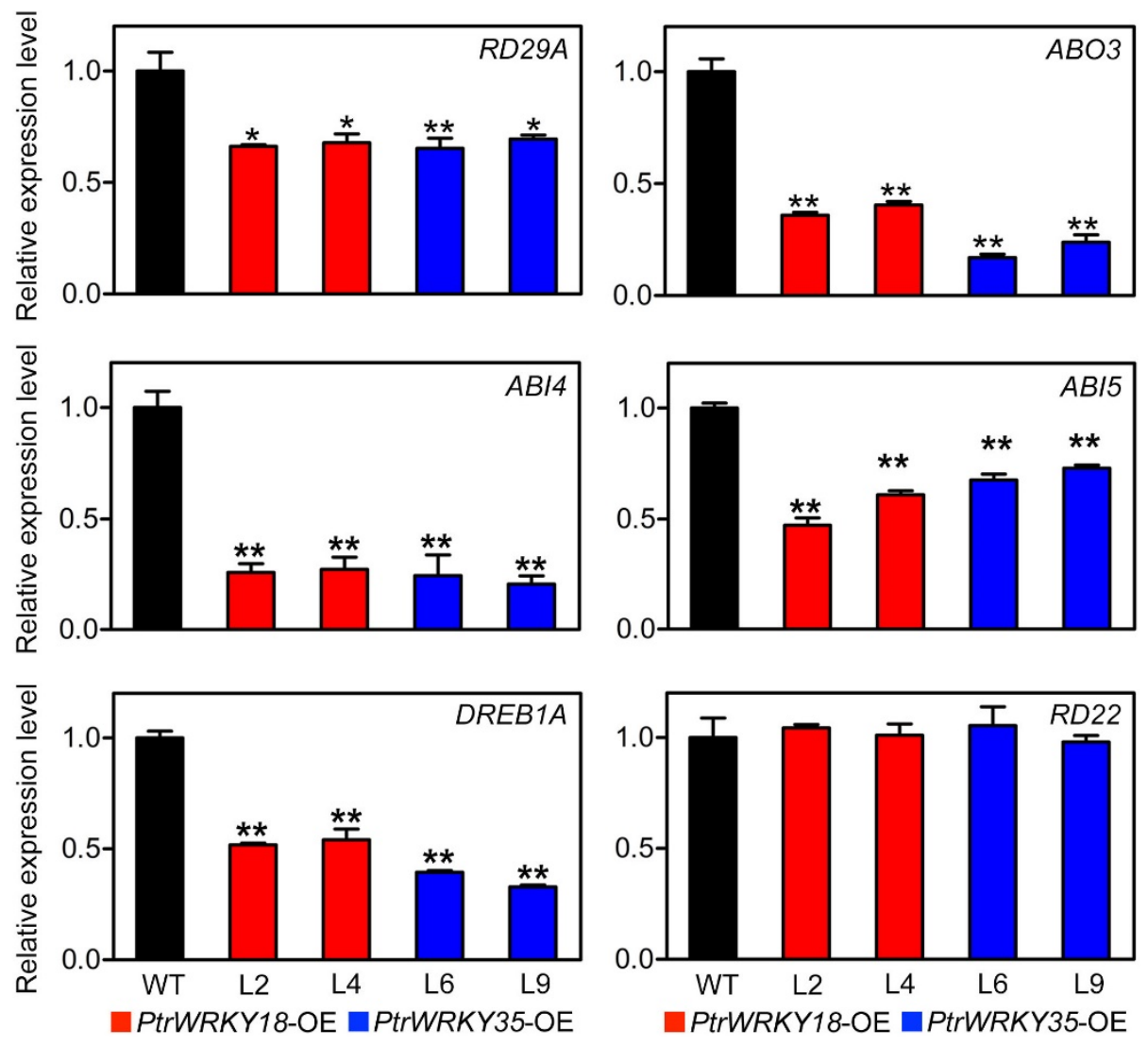

Figure 6. Expression level of ABA-related genes in WT and plants overexpressing PtrWRKY18 or PtrWRKY35. RD29A: responsive to desiccation 29A, ABO3: ABA overly sensitive 3, ABI4/5: ABA insensitive 4/5, DERB1A: dehydration response element $B 1 A, R D 22$ : responsive to dehydration 22 . Three repeats of biological replicates were performed for each gene, and each experiment contained three technical replicates. Actin was used as a reference for normalization, error bars represent $\mathrm{SD}\left({ }^{*} p<0.05\right.$; $\left.{ }^{* *} p<0.01\right)$.

In short, PtrWRKY18 and PtrWRKY35 can be induced by various stresses, and have a variety of functions in response to environmental stress. Heterologous overexpression of PtrWRKY18 and PtrWRKY35 in Arabidopsis not only leads to enhanced JA-induced defense against necrotrophic pathogens and weakened SA-induced defense against biotrophic pathogen, but also affects drought tolerance via the ABA-independent signaling pathway.

\section{Discussion}

WRKYs have been widely reported to play key and various roles in regulating plant growth and development, including somatic embryogenesis [33], seed coat pigmentation and development [34,35], trichome development [36], leaf senescence [37,38], as well as various abiotic and biotic stress 
responses [39,40]. Recently, most of the studies about WRKYs are focused on abiotic and biotic stress responses, especially at the transcriptional level [41-43]. A great deal of results demonstrate that WRKYs are key regulators in basal defense responses of many plant species, such as Arabidopsis, rice, strawberry, grapevine, poplar, and tobacco [44-50]. Meanwhile, many WRKY proteins are involved in SA-mediated defense against biotrophic pathogens. In Arabidopsis, 49 members of 72 WRKYs can be induced by SA treatment or significantly regulated through pathogenic infections [51]. AtWRKY70 has been demonstrated to be an activator of the SA signal pathway to reduce resistance to Alternaria brassicicola and enhance resistance to Erysiphe cichoracerum [52,53]. JA and SA often play antagonistically roles in defense responses, and some WRKY proteins also take part in JA-mediated defense against herbivorous insects or necrotrophic pathogens. CaWRKY27 and CaWRKY 40 act as positive regulators in tobacco resistance against Ralstonia solanacearum through the regulation of SA-, JA-, and ethylene-mediated signaling pathways [54,55]. In Arabidopsis, overexpression of AtMYB44 can reduce the plant defense response to the necrotrophic pathogen Alternaria brassicicola and enhance resistance to the biotrophic pathogen Pst DC3000 via regulating AtWRKY70 expression and modulating antagonistic interaction between the SA and JA signaling pathway [56]. It has also been proven that the double mutants of Atwrky18/Atwrky40 and Atwrky18/Atwrky60 showed a higher resistance to P. syringae, but were more susceptible to B.cinerea $[15,16]$.

Recently, growing evidence shows that WRKYs are also involved in the ABA signaling pathway $[29,57,58]$. In Arabidopsis, AtWRKY18 and AtWRKY60 have positive effects on plant ABA sensitivity, and inhibit seed germination and root growth, whereas AtWRKY 40 has a negative effect on plant ABA sensitivity and promotes the seed germination and root growth [15]. Constitutive expression of GhWRKY17 in tobacco remarkably reduced plant drought and salt tolerance, and enhanced plant ABA sensitivity to inhibit seed germination and root growth [58].

\subsection{SA, JA, and ABA All Play Important Roles in Regulating the Expression of PtrWRKY18 and PtrWRKY35}

In our previous studies, two poplar WRKY transcription factors, PtrWRKY18 and PtrWRKY35, have been identified and isolated [22]. They have been demonstrated to be the homologous genes of AtWRKY18, AtWRKY40, and AtWRKY60, and can activate pathogenesis-related genes to increase resistance to the biotrophic pathogen Melampsora via SA-mediated signal pathway in poplar [22]. Their homologous genes in Arabidopsis also have vital effects on plant ABA sensitivity and drought tolerance, and inhibit seed germination and root growth [15]. Therefore, we have a hypothesis that PtrWRKY18 and PtrWRKY35 are multifunctional transcription factors, and participate extensively in plant biotic and abiotic stress responses.

To test the hypothesis, various treatments (SA, JA, P. syringae, B. cinerea, and ABA) were applied to the leaves of transgenic Arabidopsis containing GUS report genes. Expression levels of PtrWRKY18 and PtrWRKY 35 were significantly up-regulated under the treatments of SA, JA, P. syringae, and B. cinerea and down-regulated under the ABA treatment (Figure 1). The results suggested that PtrWRKY18 and PtrWRKY35 were regulated by multiple hormones.

\subsection{PtrWRKY18 and PtrWRKY35 Play Antagonistic Roles in JA and SA Signaling Pathway}

To study the versatility of PtrWRKY18 and PtrWRKY35, we heterologous overexpressed them in Arabidopsis. B. cinerea was introduced to activate JA-mediated defense against necrotrophic pathogens. As expected, overexpression of PtrWRKY18 or PtrWRKY35 could activate pathogenesis-related genes to increase resistance to B. cinerea in Arabidopsis (Figure 2).

To investigate the effects of heterologous overexpression of PtrWRKY18 or PtrWRKY35 on the SA signaling pathway, the transgenic Arabidopsis were treated with P. syringae. Surprisingly, overexpression of them in Arabidopsis inhibited the expression of marker genes in the SA signaling pathway, thereby reducing plant resistance to P. syringae (Figure 3).

Those results suggest that heterologous expression of PtrWRKY18 or PtrWRKY35 in Arabidopsis activated the JA signaling pathway (Figure 2), but inhibited the SA signaling pathway (Figure 3). 
The results were consistent with antagonistic effects of SA and JA in many aspects of plant growth and development, including defense responses [24].

In our previous study, we demonstrated that PtrWRKY18 or PtrWRKY35 played positive roles in the P. tomentosa SA signaling pathway [22]. Meanwhile, we have not discovered their function in the JA signaling pathway of $P$. tomentosa. Combined with the research in this paper, we can conclude that the distinct functions of PtrWRKY18 and PtrWRKY35 in Arabidopsis and poplar may owing to species difference.

3.3. PtrWRKY18 and PtrWRKY35 Play Roles in ABA Signal Perception, and Can Regulate the Tolerance to Osmotic Stress through Stomatal Movement

Generally, ABA acts as an important hormone to deal with various biotic and abiotic stresses. High salinity and drought stimulation can promote its accumulation $[25,26]$. In this study, we found that the expression of PtrWRKY18 and PtrWRKY35 was inhibited by ABA signals and that, compared with WT, the germination rate and root development of Arabidopsis were suppressed (Figure 4). The results suggest that they may negatively regulate ABA signaling.

We have shown that overexpression of PtrWRKY18 and PtrWRKY 35 remarkably reduced the tolerance of Arabidopsis to high salinity and drought treatment (Figure 5A,B). As a major indicator of ABA-mediated osmotic stress response, the stomatal aperture of guard cells from transgenic plants was larger than that of WT under ABA treatment (Figure 5C,D). Some key transcription factors in ABA pathway were also down-regulated in transgenic plants (Figure 6). The results told us that PtrWRKY18 and PtrWRKY35 indeed played negative roles in the ABA-dependent signaling pathway.

Taken together, PtrWRKY18 and PtrWRKY35, with similarities to AtWRKY18, AtWRKY40, and AtWRKY60, were multifunctional transcription factors in plant resistance. In plant immunity, PtrWRKY18 and PtrWRKY35 not only play important roles in the SA-mediated pathway [22], but also function in regulating the JA signaling pathway. Meanwhile, they have a negative effect on ABA sensitivity, and reduce plants' tolerance to osmotic stress.

To better understand the respective functions of $\operatorname{Pt} W R K Y 18$ and PtrWRKY35, overexpression of PtrWRKY18 and PtrWRKY35 in AtWRKY18, AtWRKY40, and AtWRKY60 single, double, or triple mutants may be viable methods. A more detailed functional analysis of PtrWRKY18 and PtrWRKY35 will help us improve poplar trees through genetic engineering techniques, and cultivate various poplar varieties that can adapt to extreme environments.

\section{Materials and Methods}

\subsection{Plant Material and Treatments}

As described in our previous study (Jiang et al. 2007), the full-length coding sequences of WRKY18 and WRKY35 were amplified from the cDNA that was reversely transcribed from the mRNA of P. trichocarpa. The amplified cDNA fragments were subsequently constructed in the PCXSN vector under the control of the $35 S$ promoter for overexpression. The resulting constructs were genetically transformed into Arabidopsis using the method of agrobacterium-mediated floral dip. The positive transgenic plants of T1 generation were screened out by hygromycin resistance and PCR genotyping, and pollinated for T2 generation. The produced T2 seeds were germinated on hygromycin-containing MS medium, and calculated for the ratio of survival/death (3:1) to identify the transgenic plants of single insertion. The single copy-inserted transgenic plants were pollinated for T3 generation. The T3 seeds were germinated on the medium supplemented with hygromycin, and the lines, the seedlings of which all survived, were considered as the homozygotes. The highly expressed transgenic lines were detected by RT-PCR using the primer used previously [22].

The seeds of WT Col-0, homozygous PtrWRKY18, and PtrWRKY35 overexpressing lines were kept at $4{ }^{\circ} \mathrm{C}$ for 3 days before being placed on MS medium [59] supplemented with $3 \%(w / v)$ sucrose. After 10 days of germination on MS plates, the seedlings were transferred into soil and developed in a 
growth incubator at $22{ }^{\circ} \mathrm{C}$ under long-day conditions ( $16 \mathrm{~h} \mathrm{light} / 8 \mathrm{~h}$ dark), with $80 \%$ relative humidity for further analysis.

For hormonal treatments, SA ( $5 \mathrm{mM}$ in water), JA [1 $\mathrm{mM}$ in $0.1 \%(v / v)$ ethanol], and ABA [25 $\mu \mathrm{M}$ in $0.1 \%(v / v)$ ethanol] solutions were sprayed on whole plants, respectively. The water or the solution of $0.1 \%(v / v)$ ethanol without any hormones was used as the mock control. Each treatment was performed for more than four biological replicates. The treated plants were covered with a transparent film sheet for $24 \mathrm{~h}$. Then, leaves were detached for GUS staining. Inoculation of Botrytis cinerea (B. cinerea) and Pseudomonas syringae pv. tomato DC3000 (P. syringae) was performed as described previously [60].

In order to observe the difference in sensitivity to ABA during seed germination, seeds were evenly placed on MS medium supplemented with 3\% $(w / v)$ sucrose and $0.3 \mu \mathrm{M}$ ABA. Statistical germination rates were calculated from 1 to 9 days after the earliest germination (DAG). For root length statistics, seeds were placed on square petri dish containing normal MS medium supplemented with $3 \%(w / v)$ sucrose and MS medium supplemented with 3\% $(w / v)$ sucrose and $10 \mu \mathrm{M}$ ABA, respectively. Root lengths were measured after 8 days of vertical culture.

For $\mathrm{NaCl}$ and drought treatment, WT and transgenic plants were germinated simultaneously on the culture dishes and then planted in soil. After 3 weeks of growth, those plants were cultivated in $300 \mathrm{mM} \mathrm{NaCl}$ treatment for 7 days or natural drought conditions (water was withheld) for 14 days, respectively. For the death ratio, those plants that suffered drought were watered again for 7 days. Each experiment was repeated three times and at least 10 plants from the individual lines were used in each experiment.

\subsection{GUS Staining}

The 1500 bp promoter fragments of PtrWRKY18 and PtrWRKY35 were amplified from the genomic DNA of $P$. trichocarpa, and ligated into pCXGUS-P vector to drive the GUS ( $\beta$-glucuronidase) reporter gene to obtain the vectors of PtrWRKY18pro: GUS and PtrWRKY35pro: GUS, respectively. The constructs were transferred into Agrobacterium tumefaciens GV3101, and transformed into Arabidopsis using the floral dip method. Single-inserted positive plants were selected according to the method mentioned above. Two-week-old homozygous transgenic plants of T3 generation harboring a single copy of insertion were treated with hormones or inoculated with fungal pathogens. Then, the leaves were detached to detect GUS activity via histochemical staining. GUS staining was performed in X-Gluc solution ( $2 \mathrm{mM} \mathrm{X-Gluc,} 0.1 \mathrm{M}$ sodium phosphate buffer (pH 7), $2 \mathrm{mM} \mathrm{K}_{4} \mathrm{Fe}(\mathrm{CN})_{6}, 2 \mathrm{mM} \mathrm{KNaPO} 4$ (pH 7) $10 \mathrm{mM} \mathrm{EDTA}, 2 \mathrm{mM} \mathrm{K}_{3} \mathrm{Fe}(\mathrm{CN})_{6}$, and $0.2 \%$ Triton X-100) under a tubes at $37^{\circ} \mathrm{C}$ for $4 \mathrm{~h}$ in the dark. Chlorophyll was removed using $70 \%(v / v)$ ethanol. At least five leaves independent transgenic lines of WRKY18pro: GUS or WRKY35pro: GUS as biological replicates for each treatment were used for GUS staining.

\subsection{Chlorophyll Content Detection}

Extraction and measurement of chlorophyll were performed as previously described [60]. Briefly, $0.2 \mathrm{~g}$ of leaves was fully grinded with a small amount of calcium carbonate powder and quartz sand in $3 \mathrm{~mL}$ acetone. All homogenates were combined in $80 \%(v / v)$ acetone and filtered with filter paper. Finally, the filtrate was added to $100 \mathrm{~mL}$ with $80 \%(v / v)$ acetone, and then the absorbance of supernatant at 663 (A663) and 645 (A645) nm was determined using UV/visible spectrophotometer Model DU800 (Shimadzu Corporation, Kyoto, Japan). The total chlorophyll (C) content was calculated using the formula below: $\mathrm{C}(\mathrm{mg} / \mathrm{g})=(20.2 \mathrm{~A} 645+8.02 \mathrm{~A} 663) / 2$. The measurements were repeated for three biological replicates of each transgenic line.

\subsection{Growth State Detection of P. syringae in Plants}

The growth situation of $P$. syringae in plants was indicated by the number of colonies in the leaves. The injected leaves of Arabidopsis were diluted and then spread on KB (King's B) medium, and the number of $P$. syringae colonies on the flat plate was counted after three days. 


\subsection{Stomatal Movement Assay Response to ABA}

Rosette leaves of two-week-old plants were floated in buffer for total opening of stomatal containing $10 \mathrm{mM}$ MES-Tris, $50 \mathrm{mM} \mathrm{KCl}$, pH 6.5, and exposed to light for $2.5 \mathrm{~h}$. Subsequently, ABA was added to the solution up to $25 \mu \mathrm{M}$. After ABA treatment for $2.5 \mathrm{~h}$, stomatal length and width were measured under the microscope, and the ratio of stomatal width to length was used as an indicator of stomatal opening. Thirty biological replicates were conducted.

\subsection{Gene Expression Analysis}

For quantitative real time PCR (qRT-PCR) analysis, total RNA from fresh tissues was extracted using RNA RNeasy Plant Mini Kit (Qiagen, Hilden, Germany) and treated with RNase-free DNase (TaKaRA, Dalian, China). Samples from at least three plants were pooled for analysis. The quality or integrity of RNA was checked by agarose gel electrophoresis and P100 spectrophotometer (Pultton, Ann Arbor, MI, USA). The criteria of high-quality total RNA include the following: (1) sharp distinct $28 \mathrm{~S}$ and $18 \mathrm{~S}$ rRNA bands, with the $28 \mathrm{~S}$ band approximately twice as intense as the $18 \mathrm{~S}$ band; (2) the value of D260/OD280 between 1.9 and 2.0; and (3) no detected genomic DNA band. The qualified RNA samples were reversely transcribed using RT-AMV (Avian Myeloblastosis Virus) transcriptase (TaKaRa, Dalian, China). PCR amplification was performed for CDS of a random gene containing an intron to exclude DNA contamination. Subsequently, qRT-PCR was performed in a volume of $25 \mu \mathrm{L}$ containing $12.5 \mu \mathrm{L}$ of SYBR Premix ExTaq TM (TaKaRa, Dalian, China). ACTIN2 rRNA was used as an internal control. Five biological replicates of each sample and three technical replicates of each biological experiment were conducted. Primers used for qRT-PCR were listed in Table S1.

\section{Conclusions}

In the research, we isolated two WRKY TF genes PtrWRKY18 and PtrWRKY35 from Populus trichocarpa and overexpressed them in Arabidopsis. The results indicated that they play roles in antagonistic regulation on pathogen resistance and abiotic stress tolerance via variable JA, SA, and ABA pathways. However, the molecular mechanism and crosstalk of hormonal pathways remain unclear. Future work is needed to better understand how WRKY transcription factors participate in pathogen resistance and abiotic stress tolerance via several hormonal pathways together.

Supplementary Materials: The following are available online at http://www.mdpi.com/1422-0067/21/15/5440/s1, Table S1: The list of primers used in real-time quantitative PCR reactions.

Author Contributions: K.L. and C.X. conceived the research; L.G., Y.J., and C.L. performed the experiments; L.G. and C.L. drafted the manuscript; C.X. and K.L. corrected the manuscript. All authors have read and agreed to the published version of the manuscript.

Funding: This work was supported by the National Natural Science Foundation of China (31870175 and31870657) and the Chongqing Youth Top Talent Program (CQYC201905028).

Conflicts of Interest: The authors declare that the research was carried out without any potential conflict of interest.

\section{References}

1. Miller, G.; Schlauch, K.; Tam, R.; Cortes, D.; Torres, M.A.; Shulaev, V.; Dangl, J.L.; Mittler, R. The Plant NADPH Oxidase RBOHD Mediates Rapid Systemic Signaling in Response to Diverse Stimuli. Sci. Signal. 2009, 2, ra45. [CrossRef] [PubMed]

2. Chen, L.; Song, Y.; Li, S.; Zhang, L.; Zou, C.; Yu, D. The role of WRKY transcription factors in plant abiotic stresses. Biochim. Biophys. Acta (BBA) Bioenerg. 2012, 1819, 120-128. [CrossRef] [PubMed]

3. Fujita, M.; Fujita, Y.; Noutoshi, Y.; Takahashi, F.; Narusaka, Y.; Yamaguchi-Shinozaki, K.; Shinozaki, K. Crosstalk between abiotic and biotic stress responses: A current view from the points of convergence in the stress signaling networks. Curr. Opin. Plant Biol. 2006, 9, 436-442. [CrossRef] [PubMed]

4. Phukan, U.J.; Jeena, G.S.; Shukla, R.K. WRKY Transcription Factors: Molecular Regulation and Stress Responses in Plants. Front. Plant Sci. 2016, 7. [CrossRef] 
5. Rushton, P.J.; Somssich, I.E.; Ringler, P.; Shen, Q.J. WRKY transcription factors. Trends Plant Sci. 2010, 15, 247-258. [CrossRef]

6. Zhu, Z.; Shi, J.; Cao, J.; He, M.; Wang, Y. VpWRKY3, a biotic and abiotic stress-related transcription factor from the Chinese wild Vitis pseudoreticulata. Plant Cell Rep. 2012, 31, 2109-2120. [CrossRef]

7. Jiang, W.; Yu, D. Arabidopsis WRKY2 transcription factor mediates seed germination and postgermination arrest of development by abscisic acid. BMC Plant Biol. 2009, 9, 96. [CrossRef]

8. Rinerson, C.I.; Rabara, R.C.; Tripathi, P.; Shen, Q.J.; Rushton, P.J. The evolution of WRKY transcription factors. BMC Plant Biol. 2015, 15, 66. [CrossRef]

9. Oh, S.-K.; Yi, S.Y.; Yu, S.H.; Moon, J.; Park, J.M.; Choi, D. CaWRKY2, a chili pepper transcription factor, is rapidly induced by incompatible plant pathogens. Mol. Cells 2006, 22, 58-64.

10. Karim, A.; Jiang, Y.; Guo, L.; Ling, Z.; Ye, S.; Duan, Y.; Li, C.; Luo, K. Isolation and characterization of a subgroup IIa WRKY transcription factor PtrWRKY40 from Populus trichocarpa. Tree Physiol. 2015, 35, 1129-1139. [CrossRef]

11. Zheng, Z.; Mosher, S.L.; Fan, B.; Klessig, D.F.; Chen, Z. Functional analysis of Arabidopsis WRKY25 transcription factor in plant defense against Pseudomonas syringae. BMC Plant Biol. 2007, 7, 2. [CrossRef] [PubMed]

12. Lai, Z.; Vinod, K.; Zheng, Z.; Fan, B.; Chen, Z. Roles of Arabidopsis WRKY3 and WRKY4 Transcription Factors in Plant Responses to Pathogens. BMC Plant Biol. 2008, 8, 68. [CrossRef] [PubMed]

13. Rushton, D.L.; Tripathi, P.; Rabara, R.C.; Lin, J.; Ringler, P.; Boken, A.K.; Langum, T.J.; Smidt, L.; Boomsma, D.D.; Emme, N.J.; et al. WRKY transcription factors: Key components in abscisic acid signalling. Plant Biotechnol. J. 2012, 10, 2-11. [CrossRef] [PubMed]

14. Ren, X.; Chen, Z.; Liu, Y.; Zhang, H.; Zhang, M.; Liu, Q.; Hong, X.; Zhu, J.-K.; Gong, Z. ABO3, a WRKY transcription factor, mediates plant responses to abscisic acid and drought tolerance in Arabidopsis. Plant J. 2010, 63, 417-429. [CrossRef]

15. Chen, H.; Lai, Z.; Shi, J.; Xiao, Y.; Chen, Z.; Xu, X. Roles of arabidopsis WRKY18, WRKY40 and WRKY60 transcription factors in plant responses to abscisic acid and abiotic stress. BMC Plant Biol. 2010, 10, 281. [CrossRef]

16. Liu, Z.-Q.; Yan, L.; Wu, Z.; Mei, C.; Lu, K.; Yu, Y.-T.; Liang, S.; Zhang, X.-F.; Wang, X.-F.; Zhang, D.-P. Cooperation of three WRKY-domain transcription factors WRKY18, WRKY40, and WRKY60 in repressing two ABA-responsive genes ABI4 and ABI5 in Arabidopsis. J. Exp. Bot. 2012, 63, 6371-6392. [CrossRef]

17. Xie, Z.; Zhang, Z.-L.; Zou, X.; Huang, J.; Ruas, P.; Thompson, D.; Shen, Q.J. Annotations and Functional Analyses of the Rice WRKY Gene Superfamily Reveal Positive and Negative Regulators of Abscisic Acid Signaling in Aleurone Cells. Plant Physiol. 2005, 137, 176-189. [CrossRef]

18. Huang, Y.; Feng, C.-Z.; Ye, Q.; Wu, W.-H.; Chen, Y.-F. Arabidopsis WRKY6 Transcription Factor Acts as a Positive Regulator of Abscisic Acid Signaling during Seed Germination and Early Seedling Development. PLoS Genet. 2016, 12, e1005833. [CrossRef]

19. Chen, L.; Xiang, S.; Chen, Y.; Li, D.; Yu, D. Arabidopsis WRKY45 Interacts with the DELLA Protein RGL1 to Positively Regulate Age-Triggered Leaf Senescence. Mol. Plant 2017, 10, 1174-1189. [CrossRef]

20. Wang, H.; Avci, U.; Nakashima, J.; Hahn, M.G.; Chen, F.; Dixon, R.A. Mutation of WRKY transcription factors initiates pith secondary wall formation and increases stem biomass in dicotyledonous plants. Proc. Natl. Acad. Sci. USA 2010, 107, 22338-22343. [CrossRef]

21. Jiang, Y.; Duan, Y.; Yin, J.; Ye, S.; Zhu, J.; Zhang, F.; Lu, W.; Fan, D.; Luo, K. Genome-wide identification and characterization of the Populus WRKY transcription factor family and analysis of their expression in response to biotic and abiotic stresses. J. Exp. Bot. 2014, 65, 6629-6644. [CrossRef] [PubMed]

22. Jiang, Y.; Guo, L.; Ma, X.; Zhao, X.; Jiao, B.; Li, C.; Luo, K. The WRKY transcription factors PtrWRKY18 and PtrWRKY35 promote Melampsora resistance in Populus. Tree Physiol. 2017, 37, 665-675. [CrossRef] [PubMed]

23. Ahuja, I.; De Vos, R.C.; Bones, A.M.; Hall, R.D. Plant molecular stress responses face climate change. Trends Plant Sci. 2010, 15, 664-674. [CrossRef] [PubMed]

24. Cutler, S.R.; Rodriguez, P.L.; Finkelstein, R.R.; Abrams, S.R. Abscisic Acid: Emergence of a Core Signaling Network. Annu. Rev. Plant Boil. 2010, 61, 651-679. [CrossRef]

25. Clouse, S.D. Brassinosteroid/Abscisic Acid Antagonism in Balancing Growth and Stress. Dev. Cell 2016, 38, 118-120. [CrossRef]

26. Osakabe, Y.; Yamaguchi-Shinozaki, K.; Shinozaki, K.; Tran, L.P. ABA control of plant macroelement membrane transport systems in response to water deficit and high salinity. New Phytol. 2014, 202, 35-49. [CrossRef] 
27. Yoshida, T.; Mogami, J.; Yamaguchi-Shinozaki, K. ABA-dependent and ABA-independent signaling in response to osmotic stress in plants. Curr. Opin. Plant Biol. 2014, 21, 133-139. [CrossRef]

28. Amagai, A.; Honda, Y.; Ishikawa, S.; Hara, Y.; Kuwamura, M.; Shinozawa, A.; Sugiyama, N.; Ishihama, Y.; Takezawa, D.; Sakata, Y.; et al. Phosphoproteomic profiling reveals ABA-Responsive phosphosignaling pathways in Physcomitrella patens. Plant J. 2018, 94, 699-708. [CrossRef]

29. Stevenson, S.R.; Kamisugi, Y.; Trinh, C.H.; Schmutz, J.; Jenkins, J.; Grimwood, J.; Muchero, W.; Tuskan, G.A.; Rensing, S.A.; Lang, D.; et al. Genetic Analysis of Physcomitrella patens Identifies ABSCISIC ACID NON-RESPONSIVE, a Regulator of ABA Responses Unique to Basal Land Plants and Required for Desiccation Tolerance. Plant Cell 2016, 28, 1310-1327. [CrossRef]

30. Luan, S. Signalling drought in guard cells. Plant Cell Environ. 2002, 25, 229-237. [CrossRef]

31. Hovenden, M.J.; Schoor, J.K.V. Nature vs nurture in the leaf morphology of Southern beech, Nothofagus cunninghamii (Nothofagaceae). New Phytol. 2004, 161, 585-594. [CrossRef]

32. Wei, T.; Deng, K.; Liu, N.; Gao, Y.; Yang, M.; Zhang, Y.; Zheng, X.; Wang, C.; Song, W.; Chen, C. Ectopic expression of DREB transcription factor, AtDREB1A, confers tolerance to drought in transgenic Salvia miltiorrhiza. Plant Cell Physiol. 2016, 57, 1593-1609. [CrossRef] [PubMed]

33. Jin, F.; Hu, L.; Yuan, D.; Xu, J.; Gao, W.; He, L.; Yang, X.; Zhang, X. Comparative transcriptome analysis between somatic embryos (SEs) and zygotic embryos in cotton: Evidence for stress response functions in SE development. Plant Biotechnol. J. 2013, 12, 161-173. [CrossRef] [PubMed]

34. Johnson, C.S.; Kolevski, B.; Smyth, D.R. TRANSPARENT TESTA GLABRA2, a Trichome and Seed Coat Development Gene of Arabidopsis, Encodes a WRKY Transcription Factor. Plant Cell 2002, 14, 1359-1375. [CrossRef]

35. Lloyd, A.; Brockman, A.; Aguirre, L.; Campbell, A.; Bean, A.; Cantero, A.; Gonzalez, A. Advances in the MYB-bHLH-WD Repeat (MBW) Pigment Regulatory Model: Addition of a WRKY Factor and Co-option of an Anthocyanin MYB for Betalain Regulation. Plant Cell Physiol. 2017, 58, 1431-1441. [CrossRef]

36. Pesch, M.; Hülskamp, M. One, two, three ... models for trichome patterning in Arabidopsis? Curr. Opin. Plant Biol. 2009, 12, 587-592. [CrossRef]

37. Miao, Y.; Zentgraf, U. The Antagonist Function of Arabidopsis WRKY53 and ESR/ESP in Leaf Senescence Is Modulated by the Jasmonic and Salicylic Acid Equilibrium. Plant Cell 2007, 19, 819-830. [CrossRef]

38. Besseau, S.; Li, J.; Palva, E.T. WRKY54 and WRKY70 co-operate as negative regulators of leaf senescence in Arabidopsis thaliana. J. Exp. Bot. 2012, 63, 2667-2679. [CrossRef]

39. Pandey, S.P.; Somssich, I.E. The Role of WRKY Transcription Factors in Plant Immunity. Plant Physiol. 2009, 150, 1648-1655. [CrossRef]

40. Wei, W.; Hu, Y.; Han, Y.-T.; Zhang, K.; Zhao, F.-L.; Feng, J.-Y. The WRKY transcription factors in the diploid woodland strawberry Fragaria vesca: Identification and expression analysis under biotic and abiotic stresses. Plant Physiol. Biochem. 2016, 105, 129-144. [CrossRef]

41. Birkenbihl, R.P.; Diezel, C.; Somssich, I.E. Arabidopsis WRKY33 Is a Key Transcriptional Regulator of Hormonal and Metabolic Responses toward Botrytis cinerea Infection. Plant Physiol. 2012, 159, $266-285$. [CrossRef] [PubMed]

42. Chen, L.; Zhang, L.; Li, D.; Wang, F.; Yu, D. WRKY8 transcription factor functions in the TMV-cg defense response by mediating both abscisic acid and ethylene signaling in Arabidopsis. Proc. Natl. Acad. Sci. USA 2013, 110, E1963-E1971. [CrossRef] [PubMed]

43. Chi, Y.; Yang, Y.; Zhou, Y.; Zhou, J.; Fan, B.; Yu, J.-Q.; Chen, Z. Protein-Protein Interactions in the Regulation of WRKY Transcription Factors. Mol. Plant 2013, 6, 287-300. [CrossRef] [PubMed]

44. Xu, X.; Chen, C.; Fan, B.; Chen, Z. Physical and Functional Interactions between Pathogen-Induced Arabidopsis WRKY18, WRKY40, and WRKY60 Transcription Factors. Plant Cell 2006, 18, 1310-1326. [CrossRef]

45. Shen, Q.-H.; Saijo, Y.; Mauch, S.; Biskup, C.; Bieri, S.; Keller, B.; Seki, H.; Ülker, B.; Somssich, I.E.; Schulze-Lefert, P. Nuclear Activity of MLA Immune Receptors Links Isolate-Specific and Basal Disease-Resistance Responses. Science 2007, 315, 1098-1103. [CrossRef]

46. Kim, K.-C.; Lai, Z.; Fan, B.; Chen, Z. Arabidopsis WRKY38 and WRKY62 Transcription Factors Interact with Histone Deacetylase 19 in Basal Defense. Plant Cell 2008, 20, 2357-2371. [CrossRef] 
47. Schwessinger, B.; Bahar, O.; Thomas, N.; Holton, N.; Nekrasov, V.; Ruan, D.; Canlas, P.E.; Daudi, A.; Petzold, J.; Singan, V.R.; et al. Transgenic expression of the dicotyledonous pattern recognition receptor EFR in rice leads to ligand-dependent activation of defense responses. PloS Pathog. 2015, 11, e1004872.

48. Wang, M.; Vannozzi, A.; Wang, G.; Liang, Y.-H.; Tornielli, G.B.; Zenoni, S.; Cavallini, E.; Pezzotti, M.; Cheng, Z.-M. Genome and transcriptome analysis of the grapevine (Vitis vinifera L.) WRKY gene family. Hortic. Res. 2014, 1, 14016. [CrossRef]

49. Ye, S.; Jiang, Y.; Duan, Y.; Karim, A.; Fan, D.; Yang, L.; Zhao, X.; Yin, J.; Luo, K.; Li, C. Constitutive expression of the poplar WRKY transcription factor PtoWRKY60 enhances resistance to Dothiorella gregaria Sacc. in transgenic plants. Tree Physiol. 2014, 34, 1118-1129. [CrossRef]

50. Adachi, H.; Nakano, T.; Miyagawa, N.; Ishihama, N.; Yoshioka, M.; Katou, Y.; Yaeno, T.; Shirasu, K.; Yoshioka, H. WRKY Transcription Factors Phosphorylated by MAPK Regulate a Plant Immune NADPH Oxidase in Nicotiana benthamiana. Plant Cell 2015, 27, 2645-2663. [CrossRef]

51. Dong, J.; Chen, C.; Chen, Z. Expression profiles of the Arabidopsis WRKY gene superfamily during plant defense response. Plant Mol. Biol. 2003, 51, 21-37. [CrossRef] [PubMed]

52. Li, J.; Brader, G.; Palva, E.T. The WRKY70 Transcription Factor: A Node of Convergence for Jasmonate-Mediated and Salicylate-Mediated Signals in Plant Defense. Plant Cell 2004, 16, 319-331. [CrossRef] [PubMed]

53. Li, J.; Brader, G.; Kariola, T.; Palva, E.T. WRKY70 modulates the selection of signaling pathways in plant defense. Plant J. 2006, 46, 477-491. [CrossRef] [PubMed]

54. Dang, F.; Wang, Y.; She, J.; Lei, Y.; Liu, Z.; Eulgem, T.; Lai, Y.; Lin, J.; Yu, L.; Lei, D.; et al. Overexpression of CaWRKY27, a subgroup IIe WRKY transcription factor of Capsicum annuum, positively regulates tobacco resistance to Ralstonia solanacearum infection. Physiol. Plant. 2014, 150, 397-411. [CrossRef] [PubMed]

55. Dang, F.F.; Wang, Y.N.; Yu, L.; Eulgem, E.; Lai, Y.; Liu, Z.-Q.; Wang, X.; Qiu, A.-L.; Zhang, T.-X.; Lin, J.; et al. CaWRKY40, a WRKY protein of pepper, plays an important role in the regulation of tolerance to heat stress and resistance to Ralstonia solanacearum infection. Plant Cell Environ. 2013, 36, 757-774. [CrossRef] [PubMed]

56. Shim, J.S.; Jung, C.; Lee, S.; Min, K.; Lee, Y.-W.; Choi, Y.; Lee, J.S.; Song, J.T.; Kim, J.-K.; Choi, Y.D. AtMYB44 regulates WRKY70 expression and modulates antagonistic interaction between salicylic acid and jasmonic acid signaling. Plant J. 2013, 73, 483-495. [CrossRef]

57. Jiang, Y.; Liang, G.; Yu, D. Activated Expression of WRKY57 Confers Drought Tolerance in Arabidopsis. Mol. Plant 2012, 5, 1375-1388. [CrossRef]

58. Yan, H.; Jia, H.; Chen, X.; Hao, L.; An, H.; Guo, X. The Cotton WRKY Transcription Factor GhWRKY17 Functions in Drought and Salt Stress in Transgenic Nicotiana benthamiana Through ABA Signaling and the Modulation of Reactive Oxygen Species Production. Plant Cell Physiol. 2014, 55, 2060-2076. [CrossRef]

59. Murashige, T.; Skoog, F. A Revised Medium for Rapid Growth and Bio Assays with Tobacco Tissue Cultures. Physiol. Plant. 1962, 15, 473-497. [CrossRef]

60. Jiang, Y.; Guo, L.; Liu, R.; Jiao, B.; Zhao, X.; Ling, Z.; Luo, K. Overexpression of Poplar PtrWRKY89 in Transgenic Arabidopsis Leads to a Reduction of Disease Resistance by Regulating Defense-Related Genes in Salicylate- and Jasmonate-Dependent Signaling. PLoS ONE 2016, 11, e0149137. [CrossRef]

(C) 2020 by the authors. Licensee MDPI, Basel, Switzerland. This article is an open access article distributed under the terms and conditions of the Creative Commons Attribution (CC BY) license (http://creativecommons.org/licenses/by/4.0/). 